\title{
Hot and dense nuclear matter in high energy heavy ion collisions
}

\section{A. Lavagno*}

Dipartimento di Fisica, Politecnico di Torino and INFN, Sezione di Torino, Italy

E-mail: andrea.lavagno@polito.it

\begin{abstract}
We investigate the equation of state of hadronic and quark-gluon matter considering the possible formation of mixed phase at finite values of temperature and baryon density reachable in relativistic heavy ion collisions. The analysis is performed by requiring the Gibbs conditions on the global conservation of baryon number, electric charge and zero net strangeness. For hadronic phase, we study an effective relativistic mean-field model with the inclusion of $\Delta$-isobars, hyperons and lightest pseudoscalar and vector mesons degrees of freedom. For the quark sector, we employ an extended Bag model with lowest order perturbative corrections. In this context, the behavior of the hadron-quark strangeness densities in the mixed phase are analyzed.
\end{abstract}

XXth Hadron Collider Physics Symposium

November 16 - 20, 2009

Evian, France

* Speaker. 
The main goal of this contribution is to investigate the hadron-quark-gluon phase transition at finite temperature and baryon chemical potential.

Concerning the hadronic equation of state, the basic idea of the relativistic mean field model is the interaction between baryons through the exchange of mesons fields. In this context, the total Lagrangian density $\mathscr{L}$ can be written as

$$
\mathscr{L}=\mathscr{L}_{\mathrm{om}}+\mathscr{L}_{\Delta}+\mathscr{L}_{\mathrm{gfm}}
$$

where $\mathscr{L}_{\text {om }}$ stands for the full octet of lightest baryons interacting with $\sigma, \omega, \rho$ mesons fields [1]; $\mathscr{L}_{\Delta}$ corresponds to the degrees of freedom for the $\Delta$-isobars [2] and $\mathscr{L}_{\text {qfm }}$ is related to a (quasi)free gas of the lightest pseudoscalar and vector mesons with an effective chemical potential depending self-consistently from the mesons fields [3].

The implementation of hyperons degrees of freedom comes from determination of the corresponding hyperon-meson coupling constants that have been fitted to hypernuclear properties [4]. In addition, we can fix the hyperons-scalar $\sigma$ meson coupling constants to the potential depth of the corresponding hyperon in normal nuclear matter taking into account the following recent results: $U_{\Lambda}^{N}=-28 \mathrm{MeV}, U_{\Sigma}^{N}=+30 \mathrm{MeV}, U_{\Xi}^{N}=-18 \mathrm{MeV}[4]$.

Concerning the quark-gluon phase, we use an extended MIT bag model. It is well known that, using its simplest version, at moderate temperatures the deconfinement transition takes place at very large densities, if the bag pressure $B$ is fixed to reproduce the critical temperature computed in lattice QCD. On the other hand there are strong theoretical indications that at moderate and large densities (and not too large temperatures) diquark condensates can form. A phenomenological approach can therefore be based on bag constant depending on the baryon chemical potential, as proposed in Ref. [5].

At the scope of describe a hadron-quark-gluon mixed phase, we use the Gibbs formalism applied to systems where more than one conserved charge is present. In fact, because of we are going to describe the nuclear EOS at finite temperature and density with respect to strong interaction, we have to require the conservation of three "charges": baryon number, electric charge and strangeness number. Each conserved charge has a conjugated chemical potential, thus, the systems is described by three independent chemical potentials: $\mu_{B}, \mu_{C}$ and $\mu_{S}$. Therefore, the chemical potential of particle of index $i$ can be written as: $\mu_{i}=b_{i} \mu_{B}+c_{i} \mu_{C}+s_{i} \mu_{S}$, where $b_{i}, c_{i}$ and $s_{i}$ are, respectively, the baryon, the electric charge and the strangeness quantum numbers of the $i$-th hadronic species. All the thermodynamical quantities can be obtained from the baryon grand potential $\Omega_{B}$ in the standard way [6].

The structure of the mixed phase is obtained by imposing the Gibbs conditions for chemical potentials $\left(\mu_{B}^{(H)}=\mu_{B}^{(Q)}, \mu_{C}^{(H)}=\mu_{C}^{(Q)}, \mu_{S}^{(H)}=\mu_{S}^{(Q)}\right)$ and pressure $\left(P^{H}=P^{Q}\right)$. Therefore, at a given baryon density $\rho_{B}$, a given net charge fraction $Z / A$ (for example, $Z / A=0.401$ for lead-lead heavy ion collisions) and a zero net strangeness of the system $\left(\rho_{S}=0\right)$, the chemical potentials $\mu_{B}, \mu_{C}$ and $\mu_{S}$ are univocally determined by the following equations

$$
\begin{aligned}
& P^{(H)}\left(T, \mu_{B}, \mu_{C}, \mu_{S}\right)=P^{(Q)}\left(T, \mu_{B}, \mu_{C}, \mu_{S}\right), \\
& \rho_{B}=(1-\chi) \sum_{i=1}^{H} b_{i} \rho_{i}^{H}\left(T, \mu_{B}, \mu_{C}, \mu_{S}\right)+\chi \sum_{i=1}^{Q} b_{i} \rho_{i}^{Q}\left(T, \mu_{B}, \mu_{C}, \mu_{S}\right),
\end{aligned}
$$




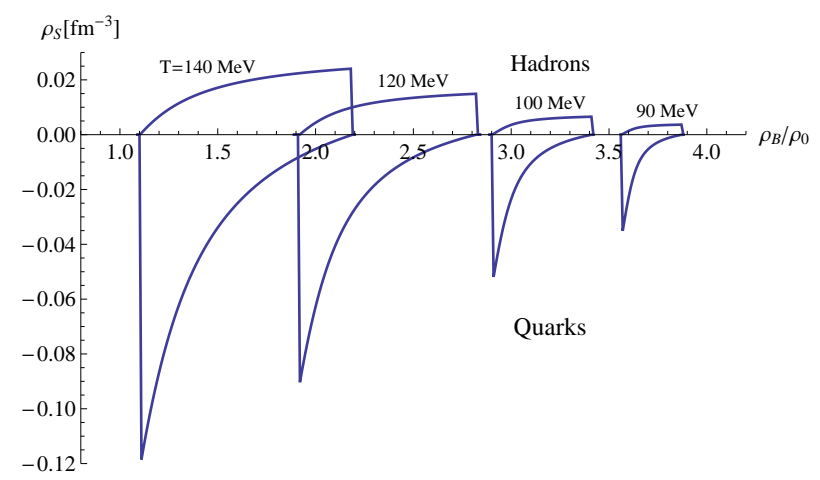

Figure 1: Net strangeness densities of the hadronic $\left(\rho_{S}>0\right)$ and quark $\left(\rho_{S}<0\right)$ components in the mixed phase region as functions of the baryon densities (in units of the nuclear saturation density $\rho_{0}$ ) and for different values of temperature.

$$
\begin{aligned}
& \rho_{C}=(1-\chi) \sum_{i=1}^{H} c_{i} \rho_{i}^{H}\left(T, \mu_{B}, \mu_{C}, \mu_{S}\right)+\chi \sum_{i=1}^{Q} c_{i} \rho_{i}^{Q}\left(T, \mu_{B}, \mu_{C}, \mu_{S}\right), \\
& \rho_{S}=(1-\chi) \sum_{i=1}^{H} s_{i} \rho_{i}^{H}\left(T, \mu_{B}, \mu_{C}, \mu_{S}\right)+\chi \sum_{i=1}^{Q} s_{i} \rho_{i}^{Q}\left(T, \mu_{B}, \mu_{C}, \mu_{S}\right),
\end{aligned}
$$

where $\rho_{i}^{H}$ and $\rho_{i}^{Q}$ are, respectively, the particle densities in the hadronic and in the quark phase and $\chi$ is the fraction volume of quark-gluon matter in the mixed phase. In this way we can find out the phase coexistence region in the $\left(T, \mu_{B}, \mu_{C}, \mu_{S}\right)$ space.

Taking into account of the above prescriptions, we are able to compute all the interesting physical quantities such as the dependence of the pressure and energy density, the fraction of produced particles at different values of temperature and baryon chemical potentials. Among the several topics of investigation, let us drive here the attention to the strangeness production in different phases of nuclear matter. In Figure 1, we report, for different values of temperature, both the net strangeness densities of hadrons and quarks in the mixed phase region (the densities are not weighed on the fraction volume $\chi$ of the phase). It is interesting to note a remarkable increasing of the net strangeness baryon-meson density and a corresponding decreasing of the strangeness quark densities at the beginning of the mixed phase. This feature becomes much more evident by increasing the temperature and could be very relevant in the phenomenological interpretation of the relativistic heavy ion collisions data.

\section{References}

[1] N.K. Glendenning, S.A. Moszkowski, Phys. Rev. Lett. 67 (1991) 2414.

[2] S. Li, G. Mao, Y. Zhou, W. Greiner, Phys. Rev. C 56 (1997) 1570.

[3] H. Müller, Nucl. Phys. A 618 (1997) 349.

[4] J. Schaffner-Bielich, A. Gal, Phys. Rev. C 62 (2000) 034311.

[5] L. Bonanno, A. Drago, A. Lavagno, Phys. Rev. Lett. 99 (2007) 242301.

[6] M. Di Toro, A. Drago, T. Gaitanos, V. Greco, A. Lavagno, Nucl. Phys. A 775 (2006) 102. 